\title{
Identification of MS-specific serum miRNAs in an international multicenter study
}

Keren Regev, MD, Brian C. Healy, PhD, Anu Paul, PhD, Camilo Diaz-Cruz, MD, Maria Antonietta Mazzola, MD, Radhika Raheja, PhD, Bonnie I. Glanz, PhD, Pia Kivisäkk, MD, PhD, Tanuja Chitnis, MD, Maja Jagodic, PhD, Fredrik Piehl, MD, PhD, Tomas Olsson, MD, Mohsen Khademi, PhD, Stephen Hauser, MD, Jorge Oksenberg, PhD, Samia J. Khoury, MD, Howard L. Weiner, MD, and Roopali Gandhi, PhD

Neurol Neuroimmunol Neuroinflamm 2018;5:e491. doi:10.1212/NXI.0000000000000491

\section{Abstract}

\section{Objective}

To identify circulating microRNAs (miRNAs) linked to disease, disease stage, and disability in MS across cohorts.

\section{Methods}

Samples were obtained from the Comprehensive Longitudinal Investigation of Multiple Sclerosis (CLIMB, Boston, MA), EPIC (San Francisco, CA), AMIR (Beirut, Lebanon) as part of the SUMMIT consortium, and Stockholm Prospective Assessment of Multiple Sclerosis (Stockholm, Sweden) cohorts. Serum miRNA expression was measured using locked nucleic acid-based quantitative PCR. Four groups were compared: (1) MS vs healthy control (HC), (2) relapsing-remitting (RR) vs $H C$, (3) secondary progressive (SP) vs $H C$, and (4) RR vs SP. A Wilcoxon rank-sum test was used for the comparisons. The association between each miRNA and the Expanded Disability Status Scale (EDSS) score was assessed using the Spearman correlation coefficient. For each comparison, the $p$ values were corrected for multiple comparisons using the approach of Benjamini and Hochberg to control the false discovery rate.

\section{Results}

In the CLIMB cohort, 5 miRNAs (hsa-miR-484, hsa-miR-140-5p, hsa-miR-320a, hsa-miR-4865p, and hsa-miR-320c) showed a significant difference between patients with MS and healthy individuals; among these, miR-484 remained significant after accounting for multiple comparisons $(p=0.01)$. When comparing RRMS with HCs, hsa-miR-484 showed a significant difference $(p=0.004)$ between the groups after accounting for multiple group comparisons. When SP and HC were compared, 6 miRNAs (hsa-miR-484, hsa-miR-140-5p, hsa-miR-142-5p, hsa-miR-320a, hsa-miR-320b, and hsa-miR-320c) remained significantly different after accounting for multiple comparisons. Disability correlation analysis with miRNA provided 4 miRNAs (hsa-miR-320a, hsa-miR-337-3p, hsa-miR-199a-5p, and hsa-miR-142-5p) that correlated with the EDSS during the internal reproducibility phase. Among these, hsa-miR-337-3p was the most statistically significant miRNA that negatively correlated with the EDSS in three of the MS cohorts tested.

\section{Conclusions}

These findings further confirm the use of circulating serum miRNAs as biomarkers to diagnose and monitor disease status in MS.

\section{Classification of evidence}

This study provides Class III evidence that levels of circulating miRNAs identify patients with MS.

\author{
Correspondence \\ Dr. Gandhi \\ rgandhibwh@gmail.com
}

\section{MORE ONLINE}

$\rightarrow$ Class of Evidence

Criteria for rating therapeutic and diagnostic studies

NPub.org/coe

From the Partners Multiple Sclerosis Center (K.R., B.C.H., A.P., M.A.M., R.R., P.K., T.C., H.L.W., R.G.), Brigham and Women's Hospital; Department of Neurology, Harvard Medical School (B.C.H., C.D.-C B.I.G., T.C., H.L.W, R.G.), Ann Romney Center for Neurologic Diseases, Brigham and Women's Hospital; Biostatistics Center (B.C.H.); Massachusetts General Hospital, Boston, MA; Department of Clinical Neuroscience, Neuroimmunology Unit (M.J., F.P., T.O., M.K.), Karolinska Institute, Stockholm, Sweden; Department of Neurology (S.H., J.O.), School of Medicine, University of California, San Francisco; and Nehme and Therese Tohme Multiple Sclerosis Center (S.J.K.), Faculty of Medicine, American University of Beirut Medical Center, Lebanon. 


\section{Glossary}

AD = Alzheimer disease; AMIR = AUBMC Multiple Sclerosis Interdisciplinary Research; ALS = amyotrophic lateral sclerosis; AUBMC = American University of Beirut Medical Center; AUC = Area under curve; AUCROC $=$ area under the ROC curve; CLIMB = Comprehensive Longitudinal Investigation of Multiple Sclerosis; EPIC study = Expression, Proteomics, Imaging, Clinical study; FDR = false discovery rate; HC = healthy control; miRNA = microRNA; RA = rheumatoid arthritis; RR = relapsing-remitting; RRMS = relapsing-remitting multiple sclerosis; SPMS = secondary progressive multiple sclerosis; STOPMS II = Stockholm Prospective Assessment of Multiple Sclerosis.

MS is an autoimmune disorder that attacks the CNS. ${ }^{1,2}$ The disease course of MS is extremely variable ${ }^{3,4}$; this diversity in the disease phenotype is not well correlated with currently used biomarkers; thus, there is an urgent need for efficacious diagnostic assays for MS detection, prognostic measures of disease progression, and treatment response.

Research on circulating biomarkers has yielded some promising candidates for MS detection including glycoproteins, chemokines, antibodies, lipopeptides, and genetic polymorphisms. More recently, microRNAs (miRNAs) have been investigated as potential biomarkers in MS. ${ }^{5-8}$ miRNAs constitute particularly exciting biomarker candidates because they are very stable molecules, and their differential expression in circulating fluids (e.g., blood) has been shown to correlate with many disease states, including $\mathrm{MS}^{5}$

Recently, our laboratory identified a set of serum miRNAs as potential diagnostic MS biomarkers. ${ }^{9}$ Differential expression of 7 miRNAs in MS vs healthy controls (HCs) was validated during a 2-year comprehensive discovery and validation study. In addition, we reported 10 miRNAs that significantly correlated with patient disability status as measured by the Expanded Disability Status Scale (EDSS). ${ }^{9}$

In the current study, we tested the reproducibility of our previous findings using patients with MS and HCs from the Comprehensive Longitudinal Investigation of Multiple Sclerosis (CLIMB) cohort. In addition, we tested the transportability of our results in MS samples collected from three other international MS collections including the SUMMIT consortium (San Franciso and Beirut) and the STOPMS II study (Stockholm).

This study design using patient samples from multiple centers $(\mathrm{n}=259)$ was implemented to identify the most promising serum miRNA biomarker candidates for MS diagnosis and disability status.

\section{Methods}

\section{Study design}

The study involved multiple phases: (1) discovery, (2) validation, (3) reproducibility, and (4) transportability phases (figure e-1, links.lww.com/NXI/A63). The first 2 phases of the study have been described previously. ${ }^{9}$ These phases are summarized as follows:
1. Discovery: 652 miRNAs were measured in participants from 3 groups: RRMS $(n=7)$, SPMS $(n=9)$, and HC $(\mathrm{n}=20)^{9}$

2. Validation: based on the findings observed in the discovery phase, 191 miRNAs were measured in a larger validation set of participants from CLIMB patients: RRMS $(n=29)$, SPMS $(n=19)$, and HC $(n=$ 30 ), including an additional cohort of participants with either other inflammatory diseases or other neurodegenerative diseases. ${ }^{9}$ This phase was used as the training set for a multivariate approach using predictive models.

3. Reproducibility phase: 73 miRNAs showing promise to serve as biomarkers ${ }^{9}$ were further analyzed in a reproducibility phase with RRMS $(\mathrm{n}=24)$, SPMS $(\mathrm{n}=18)$, and HC $(\mathrm{n}=30)$ from the CLIMB cohort. This phase was used as the test set for the predictive models that were designed in the validation (training phase).

4. Transportability phase: the 73 miRNAs from the reproducibility phase were further analyzed in 3 external cohorts with study participants from 3 MS centers worldwide: RRMS ( $\mathrm{n}=91)$, SPMS $(\mathrm{n}=33)$, and HC $(\mathrm{n}=58)$.

\section{Patient selection}

For the reproducibility phase, a new group of participants from the CLIMB cohort was identified. In addition to this new group of CLIMB participants, 3 external cohorts were included in the study. The first cohort was from the AUBMC-Multiple Sclerosis Interdisciplinary Research cohort of the American University of Beirut Medical Center-Abou Haidar Neuroscience Institute; 31 patients with RRMS, 5 patients with SPMS, and 19 HCs were included in the study. The second cohort was from the EPIC (Expression/genomics, Proteomics, Imaging, Clinical) cohort of the University of California at San Francisco ${ }^{10}$; 27 patients with RRMS, 7 patients with SPMS, and $26 \mathrm{HCs}$ were included in the study. The third cohort was from the STOPMS II (Stockholm Prospective Assessment of Multiple Sclerosis) cohort of Sweden; 33 patients with RRMS, 21 patients with SPMS, and 13 HCs were included in the study.

The demographic characteristics of all 4 cohorts are provided in table 1 . None of the selected patients were on any disease-modifying treatment at the time of sample collection. 
Table 1 Patient demographics

\begin{tabular}{|c|c|c|c|}
\hline & $\begin{array}{l}\text { Relapsing- } \\
\text { remitting } \\
\text { MS }\end{array}$ & $\begin{array}{l}\text { Secondary } \\
\text { progressive } \\
\text { MS }\end{array}$ & $\begin{array}{l}\text { Healthy } \\
\text { controls }\end{array}$ \\
\hline \multicolumn{4}{|l|}{ CLIMB } \\
\hline $\mathbf{N}$ & 24 & 18 & 30 \\
\hline Age & $32.4(6.6)$ & $56.4(9.3)$ & $45.4(15)$ \\
\hline Female & $19(0.792)$ & $15(0.833)$ & $25(0.833$ \\
\hline EDSS & $0.6(0.9)$ & $5.5(1.6)$ & \\
\hline DD in $y$ & $3.9(2.4)$ & $17.2(5.9)$ & \\
\hline \multicolumn{4}{|l|}{ AMIR } \\
\hline $\mathbf{N}$ & 31 & 5 & 19 \\
\hline Age & 33.5 (10.2) & $40.2(7.8)$ & $\begin{array}{l}37.6 \\
(11.2)\end{array}$ \\
\hline Female & $21(0.677)$ & $2(0.4)$ & $12(0.632$ \\
\hline EDSS & $2.1(1.2)$ & $4.5(1.4)$ & \\
\hline DD & $4.6(4.7)$ & $15.2(11)$ & \\
\hline \multicolumn{4}{|l|}{ EPIC } \\
\hline $\mathbf{N}$ & 27 & 7 & 26 \\
\hline Age & $37.6(6.8)$ & $52.7(5.8)$ & $\begin{array}{l}45.5 \\
(10.2)\end{array}$ \\
\hline Female & $20(0.741)$ & $7(1)$ & $12(0.462$ \\
\hline EDSS & $1(0.8)$ & $5.1(1.3)$ & \\
\hline DD & $3.9(2.4)$ & $19(6)$ & - \\
\hline \multicolumn{4}{|l|}{ STOPMS II } \\
\hline $\mathbf{N}$ & 33 & 21 & 13 \\
\hline Age & $33.1(9.4)$ & $43.2(4.3)$ & $30.9(5.6)$ \\
\hline Female & $23(0.697)$ & $11(0.524)$ & $6(0.462)$ \\
\hline EDSS & $1.66(1.7)$ & $5.3(1.5)$ & \\
\hline DD & $2.2(3.4)$ & $19.6(5.2)$ & - \\
\hline
\end{tabular}

Abbreviations: AMIR = AUBMC-Multiple Sclerosis Interdisciplinary Research cohort; CLIMB = Comprehensive Longitudinal Investigation of Multiple Sclerosis; DD = disease duration; EDSS = Expanded Disability Status Scale; EPIC = Expression/genomics, Proteomics, Imaging, and Clinical cohort; STOPMS II = Stockholm Prospective Assessment of Multiple Sclerosis.

\section{Standard Protocol Approvals, Registrations, and Patient Consents}

Written informed consent was obtained from all patients, and regional ethical committees approved the study (CLIMB cohort, IRB Protocol No: 1999P010435; EPIC cohort, IRB Protocol No: 10-00104; AMIR cohort, IRB Protocol No: IM.SK1.01; and STOPMS II cohort, IRB Protocol No: 2010/2:1).

\section{Sample collection and storage}

Blood samples were collected in glass red top serum vacutainer tubes without additives (Becton, Dickinson and
Company, Franklin Lakes, NJ), and serum tubes were kept at room temperature for 30-60 minutes. Each sample was centrifuged at $2000 \mathrm{RPM}$ for 10 minutes to separate the serum and then stored at $-70^{\circ} \mathrm{C}$ until RNA extraction. Freezing of the serum occurred within 2 hours of the blood draw. Serum samples from the external cohorts were sent to Boston for further processing.

\section{RNA extraction and analysis}

RNA was isolated using the miRCURY kit (Exiqon, Waltham, MA) and converted to cDNA using a synthesis kit from Exiqon following the manufacturer's instructions. Prepared cDNAs were stored at $-20^{\circ} \mathrm{C}$ until use. LNA SYBR greenbased real-time PCR was used to detect the expression of selected miRNAs (Exiqon). Normalization was performed using the mean expression of the miRNAs with the best stability index, i.e., showing expression stability in our sample set. NormFinder software was used to calculate the stability index. We used 5 normalizing miRNAs (hsa-miR-484, hsa-miR-30e5p, hsa-miR-590-5p, hsa-let-7d-3p, and hsa-miR-15b-5p). The formula used to calculate the normalized $\mathrm{Cq}$ values is as follows: Normalized $\mathrm{Cq}=$ average $\mathrm{Cq}-$ assay $\mathrm{Cq}$.

\section{Statistical analysis}

In the CLIMB cohort, 4 groups were compared for each miRNA: (1) all MS vs HC, (2) relapsing-remitting (RR) vs HC, (3) secondary progressive (SP) vs HC, and (4) RR vs SP, and a Wilcoxon rank-sum test was used for the comparisons. For the Wilcoxon rank-sum test, participants that had no measured expression were assigned a value below the smallest available measurement so that these participants had the smallest rank for all analyses. In addition to the group comparisons, the association between each miRNA and the EDSS score was assessed using the Spearman correlation coefficient. For each comparison, the $p$ values for miRNAs that passed validation were corrected for multiple comparisons using the approach of Benjamini and Hochberg ${ }^{11}$ to control the false discovery rate (FDR).

We also compared the miRNA expression level between the groups using a proportional odds model to adjust for age and sex. The proportional odds model is a generalization of the Wilcoxon rank-sum test that allows adjustment for other variables.

In addition to the individual miRNA comparisons, prediction models were developed in the validation cohort (training set). The prediction models were built using all miRNAs that were in the same direction in the discovery and validation phases of the study using best subset regression with 3 miRNAs in the model. Three miRNAs were chosen, given the sample size limitations in the validation set (training set). The models were evaluated using the area under the ROC curve (AUCROC) in both the validation cohort (training set) and the reproducibility cohort (test set) to estimate the ability of the model to discriminate between the 2 groups. The estimated AUCROC from the reproducibility phase of the CLIMB cohort provides a more reliable estimate because data 
used in this set were not used in model building. In addition to best subset regression, several other approaches were used to build prediction models including stepwise selection, lasso, decision trees, and random forests, and the predictive performance of these approaches in our test set was assessed.

All analyses performed in the CLIMB cohort were completed in the 3 additional MS centers' cohorts during transportability phase. The miRNAs that showed significant differential expression or a significant association with the EDSS in multiple cohorts were identified. Furthermore, the AUCROC for each of the predictive models built in the CLIMB validation cohort (training set) was calculated for each external test set.

\section{Data availability}

Anonymized data not provided in the article because of space limitation will be shared upon request of other qualified investigators.

\section{Results}

\section{Reproducibility of biomarkers in the CLIMB cohort}

The miRNAs that qualified the validation of the comparison between MS and HC in the CLIMB cohort with $p<0.05$ were tested during the reproducibility phase (table 2 ). Five previously validated miRNAs, namely, hsa-miR-484, hsa-miR140-5p, hsa-miR-320a, hsa-miR-486-5p, and hsa-miR-320c, showed a significant difference, and 1 miRNA remained significant after accounting for multiple comparisons (hsa-miR484, $p=0.01$ ). When multiple miRNAs were included in a predictive model for MS vs $\mathrm{HC}$, the miRNAs included were hsa-let-7c-5p, hsa-miR-452-5p, and has-miR-484. The AUCROC for this model in the validation cohort was 0.85 , whereas the AUCROC in the reproducibility phase was 0.70 (figure e-2, links.lww.com/NXI/A63). When the other model building approaches were used, the AUCROC in the reproducibility cohort ranged from 0.67 to 0.71 .
The comparisons of the miRNAs between $\mathrm{RR}$ and $\mathrm{HC}$ are provided in (table e-1, links.lww.com/NXI/A64). In this comparison, only hsa-miR-484 showed a significant difference ( $p=0.004)$ between the groups after accounting for multiple comparisons. When best subset regression was used to select a model for this comparison, the miRNAs included in the model were hsa-miR-15b-3p, hsa-miR-451a, and hsa-miR584-5p. The AUCROC for this model in the validation set was 0.82 , whereas the AUCROC in the reproducibility phase was 0.68 (figure e-3, links.lww.com/NXI/A63). When the other model building approaches were used, the AUCROC in the validation ranged from 0.63 (decision tree) to 0.68 (best subset regression).

When SP and HC were compared (table e-2, links.lww.com/ NXI/A64), 8 miRNAs were significantly different, and 6 miRNAs (hsa-miR-484, hsa-miR-140-5p, hsa-miR-142-5p, hsamiR-320a, hsa-miR-320b, and hsa-miR-320c) were significantly different after accounting for multiple comparisons. The miRNAs included in the model selected by best subset regression were hsa-let-7c-5p, hsa-miR-320a, and hsa-miR424-5p. The AUCROC for this model in the validation was 0.99, whereas the AUCROC in the reproducibility phase was 0.71 (figure e-4, links.lww.com/NXI/A63). When the other model building approaches were used, the AUCROC in the validation ranged from 0.71 (best subsets) to 0.75 (random forests).

When RR and SP were compared (table e-3, links.lww.com/ $\mathrm{NXI} / \mathrm{A} 64$ ), no previously validated miRNAs were significantly different between the groups. When best subset regression was used to build a model, the miRNAs included in the model were hsa-miR134-5p, hsa-miR-337-3p, and hsa-miR-532-3p. The AUCROC for this model in the validation was 0.93, whereas the AUCROC in the reproducibility phase was 0.63 (figure e-5, links.lww.com/NXI/A63). When the other model building approaches were used, the AUCROC in the validation ranged from 0.53 (decision tree) to 0.72 (lasso).

Table 2 Differential expression of significant miRNAs during the reproducibility phase (MS vs healthy controls)

\begin{tabular}{|c|c|c|c|c|c|c|c|c|c|}
\hline \multirow[b]{3}{*}{ miRNA } & \multicolumn{8}{|c|}{ Reproducibility phase } & \multirow{3}{*}{$\begin{array}{l}\text { Regev et al., } 2016 \\
\text { Wilcoxon } \\
p \text { value }\end{array}$} \\
\hline & \multicolumn{2}{|c|}{ Number Expressed } & \multicolumn{2}{|c|}{ Mean Expression } & \multirow{2}{*}{$\frac{\text { Fold Change }}{\text { MS:HC }}$} & \multirow{2}{*}{$\begin{array}{l}\text { Wilcoxon } \\
p \text { value }\end{array}$} & \multirow[b]{2}{*}{ FDR value } & \multirow{2}{*}{$\begin{array}{l}\text { Adjusted } \\
p \text { value }{ }^{a}\end{array}$} & \\
\hline & MS & $\mathrm{HC}$ & MS & $\mathrm{HC}$ & & & & & \\
\hline hsa-miR-484 & 42 & 30 & -0.38 & -0.66 & 1.21 & 0.0004 & 0.01 & $<0.01$ & $<0.01$ \\
\hline hsa-miR140-5p & 42 & 30 & -2.76 & -2.41 & 0.78 & 0.01 & 0.07 & 0.01 & $<0.01$ \\
\hline hsa-miR-320a & 42 & 30 & 1.62 & 1.3 & 1.25 & 0.02 & 0.08 & 0.02 & $<0.01$ \\
\hline hsa-miR486-5p & 42 & 30 & 2.67 & 2.04 & 1.55 & 0.02 & 0.08 & 0.01 & $<0.01$ \\
\hline hsa-miR-320c & 42 & 30 & -2.51 & -2.89 & 1.30 & 0.04 & 0.12 & 0.04 & $<0.01$ \\
\hline
\end{tabular}

Abbreviations: FDR = false discovery rate; $\mathrm{HC}=$ healthy control; miRNA = microRNA.

Fifteen miRNAs were tested, and hsa-miR-320b, hsa-miR-20a-5p, hsa-miR-25-3p, hsa-miR-365a-3p, hsa-miR-941, hsa-miR-584-5p, hsa-miR-143-3p, hsa-miR140-3p, hsa-let-7c-5p, and hsa-let-7g-5p were not significant during the reproducibility phase.

Fold change was calculated as 2(differences in mean).

a Adjusted $p$ value from proportional odds model adjusting for age and sex. 
Table 3 Correlation of significant miRNAs in association with Expanded Disability Status Scale in the reproducibility phase

\begin{tabular}{|c|c|c|c|c|c|}
\hline \multirow[b]{2}{*}{ miRNA } & \multicolumn{3}{|l|}{ Reproducibility Phase } & \multicolumn{2}{|l|}{ Regev et al., 2016} \\
\hline & Spearman estimate & $p$ Value & FDR & Spearman estimate & $p$ Value \\
\hline hsa-miR-142-5p & -0.31 & 0.05 & 0.17 & -0.39 & 0.00 \\
\hline hsa-miR-199a-5p & -0.34 & 0.03 & 0.17 & -0.32 & 0.00 \\
\hline hsa-miR-320b & 0.41 & 0.01 & 0.06 & & \\
\hline hsa-miR-337-3p & -0.41 & 0.01 & 0.06 & -0.34 & 0.00 \\
\hline
\end{tabular}

Abbreviations: FDR = false discovery rate; miRNA = microRNA.

Fifteen miRNAs were tested, and hsa-miR-140-3p, hsa-miR-142-3p, hsa-miR-16-5p, hsa-miR-199a-3p, hsa-miR25-3p, hsa-miR27a-3p, hsa-miR301b, hsa-miR320a, hsa-miR-376b-3p, hsa-miR-486-5p, and hsa-miR-877-5p were not significant during the reproducibility phase.

For the disability biomarkers, the miRNAs that were significantly correlated with the EDSS in validation as published in Regev et al. $2016^{9}$ were further measured in reproducibility phase cohort. We found that 4 miRNA, hsa-miR-320b, hsa-miR-337-3p, hsa-miR199a-5p, and hsa-miR-142-5p (table 3), were significantly correlated with the EDSS in the reproducibility phase cohort.

\section{Transportability of biomarkers in external MS cohorts}

When the additional cohorts were analyzed, a modest number of miRNAs showed significant differential expression in the same direction in multiple cohorts (table 4). For the comparison of MS and HC, 5 miRNAs were found to be

Table 4 miRNAs showing significant association/differential expression during the transportability phase miRNAs

\begin{tabular}{|c|c|}
\hline & Sites with significant differential expression in MS vs HC \\
\hline hsa-let-7e-5p & $\operatorname{AMIR}(p=0.032)$, STOPMS II $(p<0.01)$ \\
\hline hsa-let-7f-5p & CLIMB $(p=0.05)$, STOPMS II $(p=0.002)$ \\
\hline hsa-miR-486-5p & CLIMB $(p=0.02)$, STOPMS II $(p=0.013)$ \\
\hline \multirow[t]{2}{*}{ hsa-miR-30e-5p } & $\operatorname{CLIMB}(p=0.013), \operatorname{EPIC}(p=0.047)$ \\
\hline & Sites with significant differential expression in RR vs. HC \\
\hline hsa-let-7e-5p & $\operatorname{AMIR}(p=0.031)$, STOPMS II $(p=0.002)$ \\
\hline \multirow[t]{2}{*}{ hsa-let-7f-5p } & CLIMB $(p=0.038)$, STOPMS II $(p=0.001)$ \\
\hline & Sites with significant differential expression in SP vs. HC \\
\hline hsa-miR-320a & CLIMB $(p=0.005)$, STOPMS II $(p=0.018)$ \\
\hline hsa-miR-320b & CLIMB $(p=0.002)$, STOPMS II $(p=0.01)$ \\
\hline hsa-miR-320c & CLIMB $(p=0.009)$, STOPMS II $(p=0.046)$ \\
\hline \multirow[t]{2}{*}{ hsa-miR-486-5p } & CLIMB $(p=0.023)$, STOPMS $\|(p=0.016)$ \\
\hline & Sites with significant correlation between miRNA and EDSS \\
\hline hsa-miR-337-3p & $\operatorname{CLIMB}(p=0.007), \operatorname{AMIR}(p=0.038), \operatorname{EPIC}(p=0.044)$ \\
\hline hsa-miR-142-5p & $\operatorname{CLIMB}(p=0.049), \operatorname{AMIR}(p=0.023)$ \\
\hline hsa-miR-199a-5p & $\operatorname{CLIMB}(p=0.03), \operatorname{AMIR}(p=0.01)$ \\
\hline hsa-miR-330-3p & $\operatorname{CLIMB}(p=0.008), \operatorname{AMIR}(p=0.022)$ \\
\hline hsa-miR-194-5p & $\operatorname{AMIR}(p=0.034), \operatorname{EPIC}(p=0.01)$ \\
\hline hsa-miR-941 & $\operatorname{AMIR}(p=0.039), \operatorname{EPIC}(p=0.05)$ \\
\hline
\end{tabular}

Abbreviations: AMIR = AUBMC-Multiple Sclerosis Interdisciplinary Research cohort; CLIMB = Comprehensive Longitudinal Investigation of Multiple Sclerosis; EDSS = Expanded Disability Status Scale; EPIC = Expression/genomics, Proteomics, Imaging, and Clinical cohort; HC= healthy control; miRNA = microRNA; RR = relapsing-remitting MS; SP = secondary progressive MS; STOPMS II = Stockholm Prospective Assessment of Multiple Sclerosis. 
differentially expressed in 2 of the 4 MS cohorts: hsa-let-7e-5p (AMIR and STOPMS II), hsa-let-7f-5p (CLIMB and STOPMS II), hsa-miR-486-5p (CLIMB and STOPMS II), and hsa-miR-30e-5p (CLIMB and EPIC) (table 4).

For the comparison of RR and HC, 2 miRNAs were differentially expressed in 2 of the 4 MS cohorts: hsa-let-7e-5p (AMIR and STOPMS II) and hsa-let-7f-5p (CLIMB and STOPMS II) (table 4). For the comparison of SP and HC, has-miR-320a, hsa-miR-320b, hsa-miR-320c, and hsa-miR486-5p were differentially expressed in the CLIMB and STOPMS II cohorts (table 4).

When comparing the 4 cohorts in terms of association with the EDSS score, a statistically significant negative correlation with hsa-miR-337-3p was observed in 3 (CLIMB, AMIR and EPIC) of the cohorts. An additional 5 miRNAs (hsa-miR-142-5p, hsa-miR199a-5p, hsa-miR-330-3p, hsa-miR-194-5p, and hsa-miR-941) showed a significant correlation in 2 of the cohorts (table 4).

When the prediction models from the CLIMB validation were applied to the transportability phase cohorts, the performance of the models was generally poor for most of the comparisons performed. The best performance was observed in the STOPMS II cohort for the SP vs HC comparisons (AUCROC $=0.73$ for best subset model and AUCROC $=0.81$ for random forests).

hsa-miR-337-3p as a marker for disability in MS The expression of hsa-miR-337-3p negatively correlated with the EDSS in the discovery set of $\mathrm{n}=85(\mathrm{rs}=-0.34 ; p=0.0002)$ and the validation phases of $\mathrm{n}=58(\mathrm{rs}=-0.30 ; p=0.02) .{ }^{9}$ These findings were further tested in another set of samples obtained from the CLIMB study (reproducibility phase) and in collaboration with 2 other international MS centers during the transportability phase (figure 1). Results from these validation studies confirmed that hsa-miR-337-3p is negatively correlated with the EDSS in 3 of 4 cohorts as summarized in figure, $\mathrm{B}$.

In addition, we found that in the CLIMB cohort SPMS patients have significantly decreased $(p=0.01)$ expression of hsa-miR337-3p compared with the patients with RRMS (figure, C). The comparison between patients with SPMS and RRMS in the other 3 international cohorts showed a similar reduction in the expression of hsa-miR-337-3p in patients with SPMS compared with those with RRMS in 3 of the 4 cohorts (EPIC, AMIR, and CLIMB) (figure e-6, links.lww.com/NXI/A63).

\section{Discussion}

In this study, we tested the differential expression of serum miRNAs and the performance of prediction models in samples from untreated patients with MS. The study aimed at testing both reproducibility in CLIMB and transportability in multiple international centers. In our reproducibility phase cohort, we validated the differential expression of hsa-miR484 in patients with MS as compared to HC (FDR < 0.05). In addition, we found an excellent performance of the prediction model including hsa-let-7c-5p and hsa-miR-452-5p discriminating MS from HC with an AUC of 0.85. hsa-miR-484 is a known factor in the regulation of neural progenitor cells and

Figure Correlation between the Kurtzke Expanded Disability Status Scale and the relative expression of hsa-miR-337-3p

A. $\operatorname{CLIMB}(\mathrm{rs}=-0.41, p=0.007)$

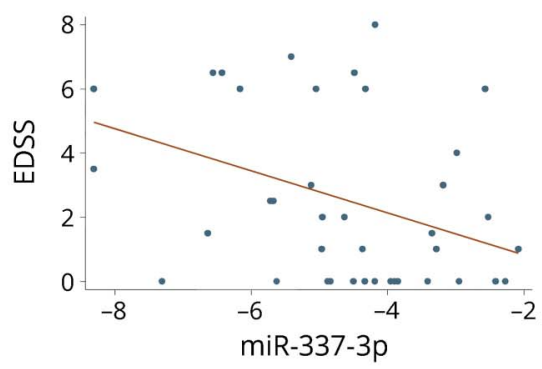

B

\begin{tabular}{|l|c|c|c|c|c|}
\hline & MS number & Mean MS & SD MS & $\begin{array}{c}\text { Spearman } \\
\text { correlation }\end{array}$ & $\boldsymbol{p}$ value \\
\hline CLIMB & 37 & -4.41 & 1.33 & -0.41 & 0.01 \\
\hline EPIC & 26 & -4.84 & 1.75 & -0.35 & 0.04 \\
\hline STOPMS II & 35 & -5.56 & 1.51 & 0.02 & 0.87 \\
\hline AMIR & 31 & -4.56 & 1.37 & -0.35 & 0.04 \\
\hline
\end{tabular}

C. CLIMB $(p=0.010)$

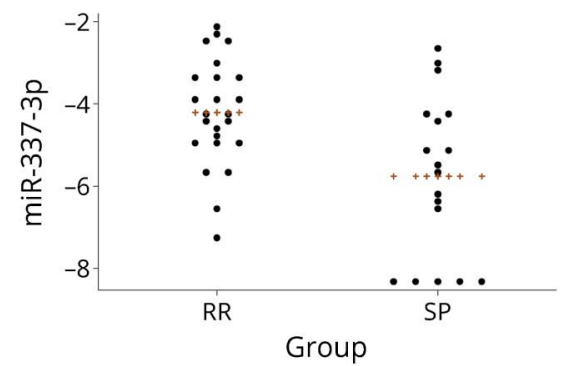

(A) Reproducibility phase: Spearman correlation between the Kurtzke EDSS and the relative expression of miR337-3p in the CLIMB cohort. (B) Transportability phase: Spearman correlation between the EDSS and the relative expression of miR-337-3p across all MS centers. Number of patients with MS expressing hsa-miR337-3p; mean MS = the mean expression value of hsa-miR-337-3p; SD MS = the SD from the mean of the expression value of hsa-miR-337-3p. (C) Differential relative expression of hsamiR-337-3p in patients with RRMS compared with patients with SPMS in the CLIMB cohort, using LNA-based qPCR. Abbreviations: AMIR = AUBMCMultiple Sclerosis Interdisciplinary Research; CLIMB = Comprehensive Longitudinal Investigation of Multiple Sclerosis; EDSS = Expanded Disability Status Scale; LNA = locked nucleic acid; $\mathrm{RR}=$ relapsing-remitting; $\mathrm{SP}=$ secondary progressive; STOPMS $\|$ = Stockholm Prospective Assessment of Multiple Sclerosis II cohort. 
its decreased expression causes dysregulated synaptogenesis and is linked to neurodevelopmental conditions including epilepsy, autism, and hyperactivity ${ }^{12}$; hence, its increased expression in MS patients' sera might actually reflect the activation of neurogenesis pathways as part of an ongoing repair process.

Although no single miRNA was identified to be differentially expressed in all 4 cohorts when comparing SPMS vs HC, we found that the expression levels of hsa-miR-486-5p, hsa-miR320a, hsa-miR-320b, and hsa-miR-320c were significantly upregulated in at least 2 independent international cohorts (table 4). Increased expression of hsa-miR-320a, hsa-miR$320 \mathrm{~b}$, and hsa-miR-320c also appears relevant to MS pathophysiology, and overexpression of miR-320 was found in MS lesions. ${ }^{13}$ Multiple hsa-miR-320 targets previously reported could be involved in MS progression and in other diseases, such as cancer. Some of these targets include CD71, MCL-1, MMP-9, NRP1, HDAC4, B-catenin, and MAPK. ${ }^{14-21}$ In this regard, the effect of hsa-miR-320 on MS pathogenesis is likely contextual, depending on the expression levels of the particular targets and the levels of this miRNA in specific cell types. One intriguing target for hsa-miR-320 is the proangiogenic NRP1 in oral cancer. ${ }^{17}$ NRP1 is highly involved in regulatory T-cell development, and it has been reported that NRP1 knockout mice exhibit a more Th17-like phenotype. ${ }^{22}$ This would suggest a pathogenic role for hsa-miR-320 in MS pathophysiology. However, when considering the other potential targets, it appears that hsa-miR-320 could also serve a protective role in MS. ${ }^{23-29}$ Of interest, differential expression of hsa-miR-320 is not unique to MS; we also found it to be significantly increased in the sera of patients with Alzheimer disease (AD) and asthma, whereas it was decreased in the sera of patients with amyotrophic lateral sclerosis (ALS) in comparison to HC samples (unpublished data and Raheja et $\mathrm{al}^{30}$ ).

We discovered that hsa-miR-337-3p and hsa-miR-199a-5p negatively correlated with patient disability (EDSS). The correlation of hsa-miR-199a-5p expression to the EDSS was observed in samples from 2 independent centers. The modest negative correlation of hsa-miR-337-3p with the EDSS was corroborated in 3 independent MS cohorts. In addition, the expression of hsa-miR-337-3p was found to be downregulated in patients with $\mathrm{AD}$ compared with healthy individuals, but was not differentially expressed in other diseases including asthma, rheumatoid arthritis (RA), and ALS (unpublished data). Our recent study on the correlation of MRI parameters to circulating miRNA expression also showed that the decreased expression of hsa-miR-337-3p is correlated with increased T1/T2 lesion load (Spearman correlation coefficient $=-0.39 ; p=$ $0.012) .{ }^{31}$ Thus, hsa-miR-337-3p is a potential biomarker candidate for MS disease progression. Of interest, it has been reported that hsa-miR-337-3p targets RAP1A in cancer. ${ }^{32}$ As RAP1A is a well-established major component of integrin activation, ${ }^{33}$ these findings points toward a potential role of hsa-miR-337-3p to serve as a biomarker for prediction of therapy response to natalizumab, an $\alpha 4 \beta 1$-integrin inhibitor. ${ }^{34}$
Some limitations encountered in this study include sample size, modest effect size of the reported findings, patient heterogeneity, and intercenter differences in patient selection and sample preparation and storage. Although we were able to confirm the transportability of several miRNAs in this international multicenter study population, we were also surprised that only few miRNAs were corroborated in all 4 centers. We believe that the inability to observe differences across all cohorts is likely attributed to these aforementioned limitations, in particular the low power of the study, especially considering the number of multiple comparisons. At the same time, we performed the analysis using all available samples rather than performing an a priori power calculation to determine the correct sample size. The heterogeneity of the population can be overcome in future studies by increasing the sample size across all comparison groups and using a standard procedure for patient's sample collection and storage. A sample size of 124 would be required to have $80 \%$ power to detect the all the differences observed in table 2 using a 2-sample $t$-test.

We were also unable to validate any differentially expressed miRNAs in the RRMS population across multiple centers. Notably, there is a higher variability in the EDSS scores of the patients with RRMS collected from multiple centers (table 1).

This is an attempt to examine the potential of miRNAs as biomarkers in an international multicenter setting for the diagnosis and correlation with disability in MS. It is also an attempt to build a prediction model in a validation phase after a training phase and a discovery phase. We found a promising candidate (hsa-miR-337-3p) to be used to identify a progression state that has great potential for translation to a clinical setting. Altogether, our work on circulating miRNA biomarkers is likely to help promote the development of novel tools for noninvasive diagnosis and therapeutic prognosis for MS.

\section{Author contributions}

R. Keren: drafting the manuscript for content, study concept and design, analysis and interpretation of data, and acquisition of data. B.C. Healy: drafting the manuscript for content, study concept, statistical analysis, and interpretation of data. A. Paul: analysis or interpretation of data, acquisition of data, and revising the manuscript for content. C. Diaz-Cruz: acquisition of data and revising the manuscript for content. M. A. Mazzola and R. Raheja: revising the manuscript for content. B. I. Glanz: design and patient selection. P. Kivisakk, T. Chitnis, M. Jagodic, F. Piehl, T. Olsson, M. Khademi, S. Hauser, J. Oksenberg, and S. J. Khoury: design, patient selection, and collection. H. L. Weiner: revising the manuscript for content, study concept and design, study supervision and coordination, and obtaining funding. R. Gandhi: drafting and revising the manuscript for content, study concept and design, analysis and interpretation of data, study supervision and coordination, and obtaining funding. 


\section{Study funding}

This study was funded by the National MS Society and by NIH common funds via NCATS UH2/UH3 grant TR000890.

\section{Disclosure}

K. Regev served on the scientific advisory boards of Merck, Sanofi, and Biogen and received travel funding from Merck, Roche, and Biogen. B.C. Healy served on the scientific advisory board of Biogen; served on the editorial board of Statistical Methods in Medical Research; and received research support from Merck Serono, Novartis, Genzyme, Verily life Sciences, and NIH. A. Paul reports no disclosures. C. DiazCruz received research support from Merck Serono and Verily Life Sciences. M.A. Mazzola reports no disclosures. R. Raheja reports no disclosures. B. Glanz received research support from Merck Serono, Verily Life Sciences, NIH, Brigham Research Institute, and NMSS. P. Kivisakk received research support from Genentech, Sanofi Genzyme, and Verily Life Sciences. T. Chitnis served on the scientific advisory boards of Novartis and Sanofi Genzyme; consulted for Biogen, Celgene, Novartis, and Sanofi Genzyme; and received research support from EMD Serono, Verily, NMSS, The Peabody Foundation, the Consortium for MS Centers, and the Guthy-Jackson Charitable Foundation. M. Jagodic served on the editorial board of Physiologic Genomics; served as an Associate Editor of Frontiers in Neurology; and received research support from AstraZeneca, The Swedish Research Council, Stockholm County Council, Karolinska Institute, The Swedish Association for Persons with Neurologic Disability, The Swedish Brain Foundation, The Swedish Medical Society, and Petrus and Augusta Hedlunds Foundation. F. Piehl served on the scientific advisory board of Praxel/Chugai and received research support from Biogen, Novartis, Genzyme, Swedish Medical Research Council, and PCORI. T. Olsson served on the scientific advisory boards of Biogen, Genzyme, Merck, and Novartis; received travel funding and/ or speaker honoraria from Novartis, Biogen, Genzyme, and Merck; served as coeditor of Current Opinion in Immunology; and received research support from Merck, Biogen, Genzyme/Sanofi, Novartis, Roche, Merck, Bayer Schering, Aventis, Biogen, AstraZeneca, The Swedish Research Council, Swedish Brain Foundation, Knut and Alice Wallenberg Foundation, Swedish Research Council, and Margaretha af Ugglas Foundation. M. Khademi reports no disclosures. S. Hauser served on the scientific advisory boards of Symbiotix, Annexon, Bionure, Molecular Stethoscope, Alector, and Neurona; received travel funding from F. Hoffmann La Roche; receives publishing royalties from McGraw-Hill Education; received writing support from F. Hoffmann La Roche; and received research support from the NIH, NMSS, and Conrad N. Hilton Foundation. H. Oksenberg served on the editorial board of Genes and Immunity and received research support from Genentech, NIH/NIAID, NIH/NINDS, University of Miami, and NMSS. S. Khoury served as an Associate Editor of Annals of Neurology, a section editor of Clinical Immunology, and editorial board member of Treatment Options in Neurology and MS Journal and received research support from Novartis. H.L. Weiner served on the scientific advisory board of The Guthy-Jackson Charitable Foundation, Teva, Biogen, Novartis, Sanofi-Aventis, Tilos, CBridge Capital, Genentech, Genzyme, vTv Therapeutics, and MedDay; consulted for Biodextris, Biogen, Novartis, Serono, Teva, Sanofi Genzme, Tilos, Tiziana Life Sciences, vTv Therapeutics, MedDay, Genentech, and CBridge Capital; and received research support from EMD Serono, Google Life Sciences, NIH, and NMSS. R. Gandhi is employed by Sanofi Genzyme and received research support from the NIH and Race to Erase MS. Full disclosure form information provided by the authors is available with the full text of this article at Neurology.org/NN.

Received January 13, 2018. Accepted in final form June 22, 2018.

\section{References}

1. Compston A, Coles A. Multiple sclerosis. Lancet 2008;372:1502-1517.

2. Weiner HL. Multiple sclerosis is an inflammatory T-cell-mediated autoimmune disease. Arch Neurol 2004;61:1613-1615.

3. Gholipour T, Healy B, Baruch NF, Weiner HL, Chitnis T. Demographic and clinical characteristics of malignant multiple sclerosis. Neurology 2011;76:1996-2001.

4. Amato MP, Zipoli V, Goretti B, et al. Benign multiple sclerosis: cognitive, psychological and social aspects in a clinical cohort. J Neurol 2006;253:1054-1059.

5. Gandhi R. miRNA in multiple sclerosis: search for novel biomarkers. Mult Scler 2015; 21:1095-1103.

6. $\mathrm{Qu} \mathrm{Z}, \mathrm{Li}$ W, Fu B. MicroRNAs in autoimmune diseases. Biomed Res Int 2014;2014: 527895 .

7. Jagot F, Davoust N. Is it worth considering circulating microRNAs in multiple sclerosis? Front Immunol 2016;7:129.

8. Sondergaard HB, Hesse D, Krakauer M, Sorensen PS, Sellebjerg F. Differential microRNA expression in blood in multiple sclerosis. Mult Scler 2013;19:1849-1857.

9. Regev K, Paul A, Healy B, et al. Comprehensive evaluation of serum microRNAs as biomarkers in multiple sclerosis. Neurol Neuroimmunol Neuroinflamm 2016;3:e267. doi: 10.1212/NXI.0000000000000267.

10. University of California SFMSET, Cree BA, Gourraud PA, et al. Long-term evolution of multiple sclerosis disability in the treatment era. Ann Neurol 2016;80:499-510.

11. Benjamini Y, Hochberg Y. Controlling the false discovery rate: a practical and powerful approach to multiple testing. J Royal Stat Soc 1995;57:289-300.

12. Fujitani M, Zhang S, Fujiki R, Fujihara Y, Yamashita T. A chromosome 16p13.11 microduplication causes hyperactivity through dysregulation of miR-484/ protocadherin-19 signaling. Mol Psychiatry 2017;22:364-374.

13. Junker A, Krumbholz M, Eisele S, et al. MicroRNA profiling of multiple sclerosis lesions identifies modulators of the regulatory protein CD47. Brain 2009;132: 3342-3352.

14. Cheng Z, Qiu S, Jiang L, et al. MiR-320a is downregulated in patients with myasthenia gravis and modulates inflammatory cytokines production by targeting mitogenactivated protein kinase 1. J Clin Immunol 2013;33:567-576.

15. Chen L, Yan HX, Yang W, et al. The role of microRNA expression pattern in human intrahepatic cholangiocarcinoma. J Hepatol 2009;50:358-369.

16. Chou J, Shahi P, Werb Z. microRNA-mediated regulation of the tumor microenvironment. Cell Cycle 2013;12:3262-3271.

17. Wu YY, Chen YL, Jao YC, Hsieh IS, Chang KC, Hong TM. miR-320 regulates tumor angiogenesis driven by vascular endothelial cells in oral cancer by silencing neuropilin 1. Angiogenesis 2014;17:247-260.

18. Fukushima T, Taki K, Ise R, Horii I, Yoshida T. MicroRNAs expression in the ethylene glycol monomethyl ether-induced testicular lesion. J Toxicol Sci 2011;36: 601-611.

19. Schaar DG, Medina DJ, Moore DF, Strair RK, Ting Y. miR-320 targets transferrin receptor 1 (CD71) and inhibits cell proliferation. Exp Hematol 2009;37:245-255.

20. Hsieh IS, Chang KC, Tsai YT, et al. MicroRNA-320 suppresses the stem cell-like characteristics of prostate cancer cells by downregulating the Wnt/beta-catenin signaling pathway. Carcinogenesis 2013;34:530-538.

21. Feng B, Chakrabarti S. miR-320 regulates glucose-induced gene expression in diabetes. ISRN Endocrinol 2012;2012:549875.

22. Solomon BD, Mueller C, Chae WJ, Alabanza LM, Bynoe MS. Neuropilin-1 attenuates autoreactivity in experimental autoimmune encephalomyelitis. Proc Natl Acad Sci U S A 2011;108:2040-2045.

23. Sellebjerg F, Krakauer M, Limborg S, et al. Endogenous and recombinant type I interferons and disease activity in multiple sclerosis. PLoS One 2012;7:e35927.

24. Mandel I, Paperna T, Miller A. Aberrant expression of the apoptosis-related proteins BAK and MCL1 in T cells in multiple sclerosis. J Neuroimmunol 2012; 244:51-56.

25. Aung LL, Mouradian MM, Dhib-Jalbut S, Balashov KE. MMP-9 expression is increased in B lymphocytes during multiple sclerosis exacerbation and is regulated by microRNA-320a. J Neuroimmunol 2015;278:185-189. 
Faraco G, Cavone L, Chiarugi A. The therapeutic potential of HDAC inhibitors in the treatment of multiple sclerosis. Mol Med 2011;17:442-447.

27. Guan H, Nagarkatti PS, Nagarkatti M. CD44 Reciprocally regulates the differentiation of encephalitogenic Th1/Th17 and Th2/regulatory $\mathrm{T}$ cells through epigenetic modulation involving DNA methylation of cytokine gene promoters, thereby controlling the development of experimental autoimmune encephalomyelitis. J Immunol 2011;186:6955-6964.

28. Xie C, Li Z, Zhang GX, Guan Y. Wnt signaling in remyelination in multiple sclerosis: friend or foe? Mol Neurobiol 2014;49:1117-1125.

29. Krementsov DN, Thornton TM, Teuscher C, Rincon M. The emerging role of p38 mitogen-activated protein kinase in multiple sclerosis and its models. Mol Cel Biol 2013;33:3728-3734.
30. Raheja R, Regev K, Healy BC, et al. Correlating serum micrornas and clinical parameters in amyotrophic lateral sclerosis. Muscle Nerve Epub 2018 Feb 21. doi: 10.1002/mus.26106.

31. Regev K, Healy BC, Khalid F, et al. Association between serum MicroRNAs and magnetic resonance imaging measures of multiple sclerosis severity. JAMA Neurol 2017;74:275-285.

32. Du L, Subauste MC, DeSevo C, et al. miR-337-3p and its targets STAT3 and RAP1A modulate taxane sensitivity in non-small cell lung cancers. PLoS One 2012;7:e39167.

33. Shimonaka M, Katagiri K, Nakayama T, et al. Rap1 translates chemokine signals to integrin activation, cell polarization, and motility across vascular endothelium under flow. J Cel Biol 2003;161:417-427.

34. Engelhardt B, Kappos L. Natalizumab: targeting alpha4-integrins in multiple sclerosis. Neurodegener Dis 2008;5:16-22. 


\title{
Neurology \\ Neuroimmunology \& Neuroinflammation
}

\author{
Identification of MS-specific serum miRNAs in an international multicenter study \\ Keren Regev, Brian C. Healy, Anu Paul, et al. \\ Neurol Neuroimmunol Neuroinflamm 2018;5; \\ DOI 10.1212/NXI.0000000000000491
}

This information is current as of August 20, 2018

\section{Updated Information \& Services}

References

Citations

Subspecialty Collections

Permissions \& Licensing

Reprints including high resolution figures, can be found at:

http://nn.neurology.org/content/5/5/e491.full.html

This article cites 34 articles, 5 of which you can access for free at: http://nn.neurology.org/content/5/5/e491.full.html\#\#ref-list-1

This article has been cited by 3 HighWire-hosted articles: http://nn.neurology.org/content/5/5/e491.full.html\#\#otherarticles

This article, along with others on similar topics, appears in the following collection(s):

Class III

http://nn.neurology.org//cgi/collection/class_iii

Multiple sclerosis

http://nn.neurology.org//cgi/collection/multiple_sclerosis

Information about reproducing this article in parts (figures,tables) or in its entirety can be found online at:

http://nn.neurology.org/misc/about.xhtml\#permissions

Information about ordering reprints can be found online:

http://nn.neurology.org/misc/addir.xhtml\#reprintsus

Neurol Neuroimmunol Neuroinflamm is an official journal of the American Academy of Neurology.

Published since April 2014, it is an open-access, online-only, continuous publication journal. Copyright

Copyright (C) 2018 The Author(s). Published by Wolters Kluwer Health, Inc. on behalf of the American

Academy of Neurology.. All rights reserved. Online ISSN: 2332-7812.

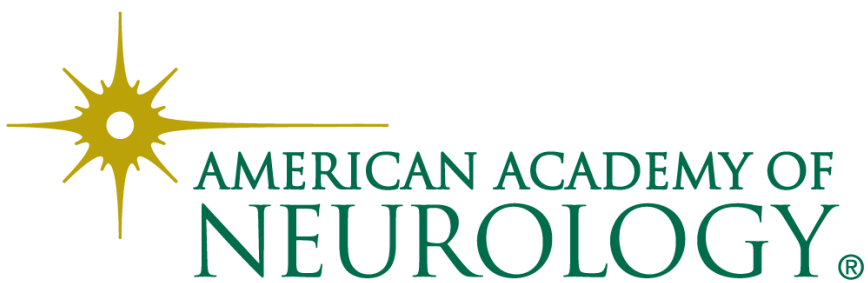

